\title{
The Nuclear Region of the Sbc Spiral Galaxy NGC 5055: A Mildly
}

\section{Active Nucleus.}

\author{
P. Pişmiş ${ }^{1}$, M. Manteiga ${ }^{2}$, A. Mampaso ${ }^{3}$, E. Recillas-Cruz ${ }^{4}$ and G. Cruz-González ${ }^{1}$ \\ ${ }^{1} U N A M$ (México) \\ ${ }^{2} \operatorname{LAEFF}$ (Spain) \\ ${ }^{3} I A C$ (Spain) \\ ${ }^{4} I N A O E$ (México)
}

\begin{abstract}
.
NGC 5055 is one of a list of nearby large spiral galaxies we have selected for a morphological and kinematic study of their bulges. CCD images are obtained at various spectral bands and in the $\mathrm{H} \alpha$ line using the $2.1 \mathrm{~m}$ reflector at San Pedro Martir observatory in México, while long-slit spectra at four different PA's are secured with the IDS spectrograph of the Isaac Newton Telescope of the observatory Roque de los Muchachos at La Palma, Spain. Some of the galaxies on our list for which observations are already performed are, aside from NGC 5055, NGC 3351, 4314, 5383 and 5915. These galaxies are not known to have active nuclei. However, we believe that activity may be a common phenomenon spanning a wide range of energetics, from the most active quasars and radio-galaxies (with jets) down to the mildest cases like our Galaxy or M31. The latter may be designated as MAGN (mildly active galactic nuclei). These ideas are treated in a review by Pişmiş (1987, Rev. Mex. Astron. Astrof. 14). The MAGN are usually nearby, and hence offer the possibility to study them in sufficient detail. It is reasonable to expect that the physical phenomenon underlying activity in galactic nuclei is one and the same, and it is essentially the global parameters such as energy at the nucleus that give rise to the different classes of AGN. Thus by studying the nearby mildly active galaxies one can construct a physically acceptable model supported by observation; such model, based on firmer grounds, can then be applied to all AGN.
\end{abstract}

NGC 5055 (Sbc) with an adopted distance of $8.2 \mathrm{Mpc}$ has a very bright bulge with a tenuous multiple arm structure around it, extending to 8 arcmin. Our velocity field and morphology of NGC 5055 lead to the following conclusions.

1. The galaxy presents a north-south asymmetry; the southern approaching side has its isophotes closer together than at the farther side. We adopt PA $101^{\circ}$ for the line of nodes. Our long-slit spectra cover the position angles: $58^{\circ}, 101^{\circ}, 112^{\circ}$ and $155^{\circ}$.

2 . The rotation curve shows a linear region around the nucleus. At PA $101^{\circ}$, the line of nodes, the amplitude is $270 \mathrm{~km} \mathrm{~s}^{-1}$ between points \pm 5 arcsec on either side of the nucleus.

3. There is high concentration of mass at the nuclear region (the bulge of the galaxy). An estimate of the mass for the region where the rotation curve is linear \pm 5 arcsec, is $1.5 \times 10^{8} \mathrm{M}_{\odot}$. Burbidge et al (1960) give a total mass of $7.6 \times 10^{10} \mathrm{M}_{\odot}$. The bulge density is thus found to be 500 times the average density of this galaxy.

Analysis of the general velocity field based on four long-slit spectra shows an asymmetry between the East and West sides, \pm 5 arcsec around the nucleus of NGC 5055, suggestive of the existence of non-circular motion. Indeed evidence is found of an outflow on the west side of the Galaxy within a cone emanating from the center towards the NW with a projected radial velocity of around $80 \mathrm{~km} \mathrm{~s}^{-1}$. Thus this galaxy shows activity at its center and is a candidate for the MAGN group.

Key words: galaxy, spiral, mild activity, MAGN

T. J.-L. Courvoisier and A. Blecha: Multi-Wavelength Continuum Emission of AGN, 454.

(C) 1994 IAU. Printed in the Netherlands. 